\title{
Saponarin content and biosynthesis-related gene expression in young barley (Hordeum vulgare L.) seedlings
}

\author{
HanGyeol Lee $\cdot$ So-Yeun Woo $\cdot$ Ji-Eun Ra $\cdot$ Kwang-Sik Lee $\cdot$ Woo Duck Seo $\cdot$ Jeong Hwan Lee
}

Received: 23 October 2019 / Revised: 13 December 2019 / Accepted: 17 December 2019

(c) Korean Society for Plant Biotechnology

\begin{abstract}
Flavonoids are widely distributed secondary metabolites in plants that have a variety biological functions, as well as beneficial biological and pharmacological activities. In barley (Hordeum vulgare L.), for example, high levels of saponarin accumulate during primary leaf development. However, the effect of saponarin biosynthetic pathway genes on the accumulation of saponarin in barley is poorly understood. Accordingly, the aim of the present study was to examine the saponarin contents and expression levels of saponarin biosynthetic pathway genes [chalcone synthase (CHS), chalcone isomerase (CHI), and UDP-Glc:isovitexin 7-O-glucosyltransferase $(O G T)$ ] during early seedling developmental and under several abiotic stress conditions. Interestingly, the upregulation of $H v C H S, H v C H I$, and $H v O G T$ during early development was associated with saponarin accumulation during later stages. In addition, exposure to abiotic stress conditions (e.g., light/dark transition, drought, and low or high temperature) significantly affected the expression of $\mathrm{HvCHS}$ and $\mathrm{HvCHI}$ but failed to affect either $H v O G T$ expression or saponarin accumulation. These findings suggested that the expression of $H v O G T$, which encodes an enzyme that catalyzes the final step of saponarin biosynthesis, is required for saponarin accumulation. Taken together, the results of the present study provide a basis for metabolic engineering in barley plants, especially in regards to enhancing the contents of useful secondary metabolites, such as saponarin.
\end{abstract}

H. G. Lee · J. H. Lee $(\bowtie)$

Division of Life Sciences, Jeonbuk National University, 567 Baekje-daero, Deokjin-gu, Jeonju, Jeollabuk-do 54896, Republic of Korea

e-mail: jhwanlee90@jbnu.ac.kr

S.-Y. Woo · J.-E. Ra $\cdot$ K.-S. Lee $\cdot$ W. D. Seo

Division of Crop Foundation, National Institute of Crop Science, Rural Development Administration, Jeollabuk-do 55365, Republic of Korea
Keywords Barley, Chalcone isomerase, Chalcone synthase, Saponarin, UDP-Glc:isovitexin 7-O-glucosyltransferase

\section{Introduction}

Barley (Hordeum vulgare L.) is one of the most important crop plants worldwide and has been cultivated since ancient times for a variety of uses, especially as animal feed, human food, and malting substrate (Honsdorf et al. 2014). However, even though the production of other major cereal crops (e.g., maize, rice, and wheat) increases continuously, barley production has declined over the past two decades. Interestingly, though, since multiple studies have reported the beneficial effects of barley leaves on human health can be attributed to potent antioxidant activities (Kamiyama and Shibamoto 2012), barley is regaining research attention as a potential functional food.

Plant secondary metabolites have been used as important source of active traditional medicine for centuries. Flavonoids, as one of the most abundant natural secondary metabolites, are found in a variety of vegetative and reproductive tissues, including leaves, roots, flowers, and fruits (Middleton 1998). Such plant-derived flavonoids possess a variety of beneficial biological and pharmacological properties, including antioxidant, cardio-protective, anti-carcinogenic, anti-inflammatory, anti-proliferative, anti-angiogenic, and estrogenic properties (Seo et al. 2013). Saponarin, for example, is a naturally occurring diglycoside flavone (apigenin6- $\mathrm{C}$-glucosyl-7-Oglucoside) that is likely responsible for the immunostimulatory, hypocholesterolemic, anti-carcinogenic, anti-inflammatory, anti-microbial, anti-protozoan, molluscicidal, and anti-oxidant properties of primary barley leaves (Cushnie and Lamb 2005; Hertog et al. 1993; Mojzisova et al. 2006; Moses et al. 2014; Seo et al. 2014).

Because polyphenolic compounds exhibit such a wide 
range of biological functions in plants, it is reasonable to question whether flavonoids could affect the yield stability of plants exposed to environmental stressors. In barley, genetic information and resources regarding flavonoid biosynthetic pathways are already available. Peukert et al. (2013), for example, partially sequenced the chalcone synthase (CHS), phenylalanine ammonia-lyase (PAL), cinnamate 4-hydroxylase (C4H), flavanone 3-hydroxylase (F3H), and dihydroflavonol 4-reductase (DFR) genes of 16 barley genotypes and developed single nucleotide polymorphism (SNP) markers. However, the genes of many other enzymes involved in flavonoid biosynthesis have yet to be identified. For instance, the genes involved in the conversion of naringenin to isovitexin (apigenin6- $C$-glucoside) have not been identified in barley (Marinova et al. 2007). Furthermore, the lack of suitable mutants and the poor characterization of existing mutant lines in barley impede the identification of new genes (JendeStrid 1993; Reuber et al. 1997).

A variety of genes involved in flavonoid biosynthesis are subject to both transcriptional and post-translational regulation (Lepiniec et al. 2006; Weisskopf et al. 2006). For example, CHS, which catalyzes the first step in flavonoid synthesis, is regulated at the levels of transcription, translation, and enzymatic activity (Block et al. 1990; Hartmann et al. 2005; Hartmann et al. 1998; Knogge and Weissenbock 1986), and CHS expression is strongly affected by environmental stimuli, such as light and elicitors (Christie and Jenkins 1996; Faktor et al. 1997; Schulze-Lefert et al. 1989; Wingender et al. 1989). In addition, a variety of genes involved in the flavonoid biosynthetic pathway are differentially expressed in a spatiotemporal manner (Hutzler et al. 1998; Schulz and Weissenbo"ck 1986; 1988). This suggests that the regulation of flavonoid biosynthetic pathways is more complicated than expected.

Even though saponarin in the flavonoid compounds of barley has highly accumulated during primary leaf development (Reuber et al. 1996), the regulation of saponarin biosynthesis has yet to be established. Accordingly, the aim of the present study was to investigate the relationship between saponarin content and the expression levels of three saponarin biosynthetic genes, namely $\mathrm{CHS}$, chalcone isomerase $(\mathrm{CHI})$, and UDP-Glc:isovitexin 7-O-glucosyltransferase (OGT; hereafter, $H v C H S, H v C H I$, and $H v O G T$ ), in young barley seedlings. The effects of abiotic stressors (e.g., light/dark transition, drought, and low or high temperature) on saponarin content and gene expression were also investigated.

\section{Materials and Methods}

\section{Database Searching and Sequence Analysis}

The sequence data of barley saponarin biosynthesis-related genes ( $H v C H S, H v C H I$, and $H v O G T$ ) were collected from the NCBI (https://www.ncbi.nlm.nih.gov/) and Phytozome (https://phytozome.jgi.doe.gov/pz/portal.html) databases. The $H v C H S, H v C H I$, and $H v O G T$ genes used in this study are listed in Table 1. For multiple sequence alignments, the nucleotide or amino acid sequences of $H v C H S$ and $H v O G T$ were performed using a ClustalW (http://www.ebi.ac.uk/ Tools/msa/clustalw2/) with default parameters.

Plant Materials and the Growth Conditions

Barley (Hordeum vulgare L.) seeds were soaked in water for $1 \mathrm{~d}$, germinated in the dark over $2 \mathrm{~d}$, transferred to soil, and then maintained in a growth room $\left(23^{\circ} \mathrm{C}, 16\right.$-h photoperiod, light intensity of $120 \mathrm{mmol} \mathrm{m} \mathrm{m}^{-2}$ ). Leaves were collected for analysis over a 12-d period.

Table 1 Saponarin biosynthesis-related genes analyzed in this study

\begin{tabular}{|c|c|c|c|c|}
\hline Gene Name & Transcript name & Chromosome localization & Direction & Designation for this study \\
\hline \multirow[t]{3}{*}{ Chalcone synthase } & HORVU2Hr1G116390.2 & $\begin{array}{l}\text { Chr2H:737966163.. } \\
737969220\end{array}$ & $\mathrm{R}$ & $H v C H S 1$ \\
\hline & HORVU2Hr1G004170.4 & $\begin{array}{l}\text { Chr2H:9398287.. } \\
9446222\end{array}$ & $\mathrm{R}$ & HvCHS2 \\
\hline & HORVU2Hr1G005220.3 & $\begin{array}{l}\text { Chr2H:11395530.. } \\
11400280\end{array}$ & $\mathrm{R}$ & $H v C H S 3$ \\
\hline Chalcone isomerase & HORVU5Hr1G112670.5 & $\begin{array}{l}\text { Chr5H:639991759.. } \\
639992903\end{array}$ & $\mathrm{~F}$ & $\mathrm{HvCHI}$ \\
\hline \multirow[t]{2}{*}{$\begin{array}{l}\text { UDP-Glc:isovitexin } \\
\text { 7-O-glucosyltransferase }\end{array}$} & HORVU7Hr1G031800.2 & $\begin{array}{l}\text { Chr7H:65071583.. } \\
65073314\end{array}$ & $\mathrm{R}$ & $H \nu O G T 1$ \\
\hline & HORVU3Hr1G110110.2 & $\begin{array}{l}\text { Chr3H:680047819.. } \\
680049439\end{array}$ & $\mathrm{R}$ & $H v O G T 2$ \\
\hline
\end{tabular}


For the light/dark transition treatment, 15-d-old barley plants that had been grown under continuous light conditions were transferred to a dark chamber at $23^{\circ} \mathrm{C}$ for 1 or $2 \mathrm{~d}$. For the drought treatment, 15-d-old barley plants were removed from the soil and air-dried on a filter paper in a growth chamber at $23^{\circ} \mathrm{C}$ for 1 or $2 \mathrm{~d}$. For the low and high-temperature treatments, 15 -d-old barley plants were placed in dark chambers at $4^{\circ} \mathrm{C}$ and $37^{\circ} \mathrm{C}$, respectively, for 1 or $2 \mathrm{~d}$. After harvesting, the samples were immediately frozen in liquid nitrogen for subsequent analysis. All experiments involved three biological replicates (independently harvested samples).

Ultra-high performance liquid chromatography (UHPLC) analysis

To prepare barley seedling extracts, $0.5 \sim 1 \mathrm{~g}$ dried barley seedlings were extracted in $20 \mathrm{~mL} 80 \%$ methanol $(\mathrm{v} / \mathrm{v})$ with shaking at room temperature for $1 \mathrm{~d}$. The saponarin contents of the extracts were measured using UHPLC with a UV detector (Dionex Ultimate 3000; Thermo Scientific, Waltham, MA, USA; Seo et al. 2014). Chromatographic separation was performed using a reversed-phase HPLC column (ACQUITY BEH C18, $2.1 \mathrm{~mm} \times 100 \mathrm{~mm}$, Waters, Milford, MA, USA) at $35^{\circ} \mathrm{C}$, with $0.1 \%$ TFA in water (A) and acetonitrile (B) as the mobile phases and with a flow rate of $0.5 \mathrm{~mL} / \mathrm{min}$. The gradient program was as follows: 0 3 min, 3\% B; 3 10 min, 3 15\% B; 10 13 min, 15 30\% B; $13 \sim 15 \mathrm{~min}, 30 \sim 50 \% \mathrm{~B}$; $15 \sim 16 \mathrm{~min}, 50 \sim 90 \% \mathrm{~B} ; 16 \sim 18$ $\min , 90 \% \mathrm{~B} ; 18 \sim 20 \mathrm{~min}, 90 \sim 3 \% \mathrm{~B}$. The injection volume was $2 \mu \mathrm{L}$, and the detection wavelength was $325 \mathrm{~nm}$. The saponarin content of each sample was identified by directly comparing the retention times to those of saponarin standards (Extrasynthese, Lyon, France). Three technical duplicates were performed for each of the three biological replicates (independently harvested samples). Statistical analyses were performed using SPSS for Windows (version 12.0; SPSS, Seoul, Korea). The student's $t$-test was used to compare mean saponarin contents.

\section{RNA Expression Analysis}

Total RNA was extracted from the samples using Trizol reagent (Invitrogen, Carlsbad, CA, USA), and the quality of the resulting RNA was assessed using a Nanodrop ND-2000 spectrophotometer (Nanodrop Technologies, Waltham, MA, USA). Only high-quality RNA samples (A260/A230 > 2.0 and A260/A280 > 1.8) were used for subsequent experiments. To check RNA expression, Reveres Transcriptionpolymerase chain reaction (RT-PCR) or real time-quantitative PCR (RT-qPCR) analyses were performed.

Complementary DNA (cDNA) was synthesized from $5 \mu \mathrm{g}$ RNA, following the protocol of the ReverTra Ace qPCR RT Master Mix kit (Toyobo, Osaka, Japan), and RT-qPCR analysis was conducted in 96-well plates using a CFX realtime system (Bio-Rad, Hercules, CA, USA), THUNDERBIRD SYBR qPCR mix (Toyobo), and gene-specific RT-qPCR primers (Table 2) were designed using QuantPrime (http:// quantprime.mpimp-golm.mpg.de/) and mRNA sequences from the NCBI GenBank database (http://www.ncbi.nlm.nih.gov/): HvCHSs (accession numbers: AK248641, AK354067, and AK357384), HvCHI (accession number: AK374952), and HvOGTs (accession numbers: AK371730 and AK375231). In accordance with the best-established RT-qPCR practices

Table 2 Oligonucleotides used for RT-PCR and RT-qPCR analysis in this study

\begin{tabular}{|c|c|c|c|}
\hline Gene name & Gene ID & Primers $\left(5^{\prime}\right.$ to $\left.3^{\prime}\right)$ & Direction \\
\hline \multirow[t]{3}{*}{ HvCHS1 } & AK248641 & TTTCATCATGGACGAGATGC & $\mathrm{F}$ \\
\hline & & TGTGGAGGACGACGGTCT & $\mathrm{R}$ \\
\hline & & CGCGACACAGACTAACAGGA & $\mathrm{R}$ \\
\hline \multirow[t]{2}{*}{$\mathrm{HvCHS} 2$} & AK357384 & TTTCATCATGGACGAGATGC & $\mathrm{F}$ \\
\hline & & TCAAACGACCACGATCTCAA & $\mathrm{R}$ \\
\hline \multirow[t]{2}{*}{$\mathrm{HvCHS3}$} & AK354067 & TTTCATCATGGACGAGATGC & $\mathrm{F}$ \\
\hline & & GGAATGCGTAAGCCAAGAGA & $\mathrm{R}$ \\
\hline \multirow[t]{2}{*}{$\mathrm{HvCHI}$} & AK374952 & AACTGTGTCGCGTACTGGAA & $\mathrm{F}$ \\
\hline & & GGGGAGTGCGTGAAGAGTAT & $\mathrm{R}$ \\
\hline \multirow[t]{2}{*}{$H v O G T 1$} & AK375231 & TGTCGAGATAGTTTGTCGGGTGTG & $\mathrm{F}$ \\
\hline & & ATCACGCCGTCGGATGGATATCTG & $\mathrm{R}$ \\
\hline \multirow[t]{2}{*}{$H v O G T 2$} & AK371730 & CAGCAGGAGGAACCTGGATA & $\mathrm{F}$ \\
\hline & & CTGGCACCAAGCAGCAGAT & $\mathrm{R}$ \\
\hline \multirow[t]{2}{*}{$H v P P 2 A A 3$} & AK251150 & ACATCCCTCTGCTGGCTAGT & $\mathrm{F}$ \\
\hline & & GCCAGTCGCTTCAAGTTGTT & $\mathrm{R}$ \\
\hline
\end{tabular}


a

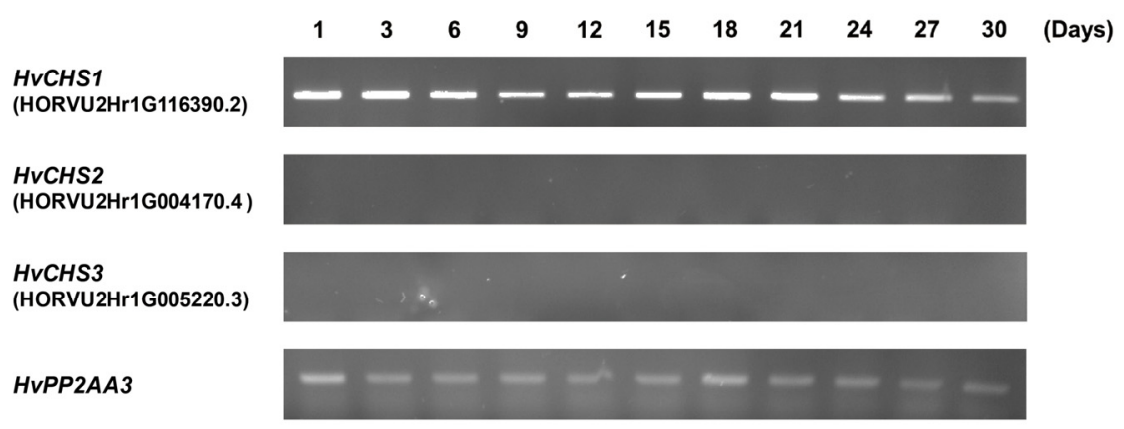

b

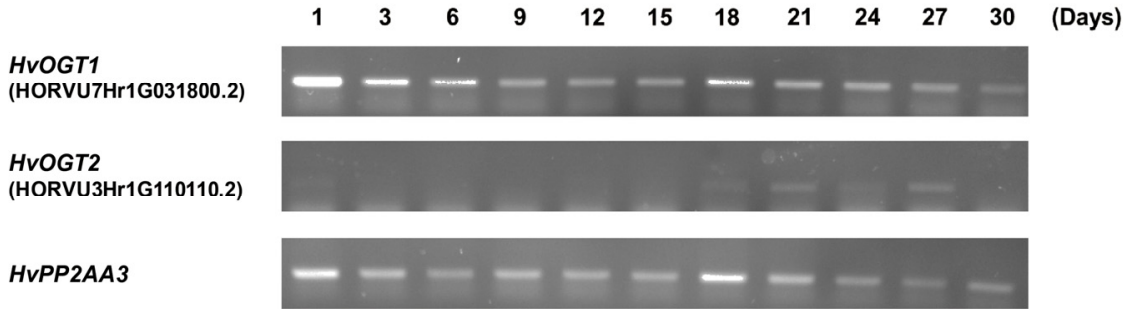

Fig. 1 Expression levels of $H v C H S 1 / 2 / 3$ and HvOGT1/2 members during barley developmental stages. (a) Expression levels of $H v C H S 1 / 2 / 3$ members. Barley plants grown at $23^{\circ} \mathrm{C}$ under long-day (LD) conditions were harvested at 1, 3, 6, 9, 12, 15, 18, 21, 24, 27, and $30 \mathrm{~d}$, and expression levels were analyzed using RT-PCR. HvPP2AA3 was used as an internal control. (b) Expression levels of HvOGT1/2 members

(Janska et al. 2013; Zhang et al. 2018), HvPP2AA3 (accession number: AK251150) was included as a stably expressed reference gene, and three technical duplicates were performed for each of the three biological replicates. To determine the relative abundance of the transcripts, the data were analyzed using Bio-Rad CFX Manager (Bio-Rad). Statistical analyses were performed using SPSS for Windows (version 12.0; SPSS, Seoul, Korea). The student's $t$-test was used to compare mean expression levels.

\section{Results and Discussion}

Identification of saponarin biosynthetic pathway genes in barley

To identify saponarin biosynthesis-related genes in barley, we searched the NCBI and Phytozome databases. The present study showed that three $H v C H S s$, one $H v C H I$, and two HvOGTS in barley genome (Table 1). Based on sequence similarity to other plant homologs, we designated the three HvCHSs as HvCHS1 [HORVU2Hr1G116390.2 (AK248641)], HvCHS2 [HORVU2Hr1G004170.4 (AK357384)], and HvCHS3 [HORVU2Hr1G005220.3 (AK354067)], and the two HvOGTs as $\mathrm{HvOGT1}$ [HORVU7Hr1G031800.2 (AK375231)] and

\section{HvOGT2 [HORVU3Hr1G110110.2 (AK371730)].}

We investigated the temporal expression patterns of three $H v C H S s$ and two HvOGTs during the developmental stages. Only HvCHS1 transcripts, but not HvCHs2 and HvCHS3 transcripts, were expressed in all developmental stages (Fig. 1a). HvOGT1 transcripts were expressed in all stages, whereas HvOGT2 transcripts were detected in later stages (from 18 to 27 d) (Fig. 1b). Furthermore, higher levels of $H v C H S 1$ and $H v O G T 1$ expression were seen in early stages (from 1 to $6 \mathrm{~d}$ ). Because higher accumulation of saponarin is found in earlier developmental stages (Reuber et al. 1996), we chose $H v C H S 1, H v C H I$, and $H v O G T 1$ for subsequent experiments.

Saponarin biosynthesis and accumulation in barley seedlings

Because saponarin accumulates to high levels as the major flavonoid compound during primary barley leaf development (Reuber et al. 1996), the expression of saponarin biosynthetic pathway genes in young barley seedlings were examined in detail. Barely plants were grown at $23^{\circ} \mathrm{C}$ under $\mathrm{LD}$ conditions and harvested after 1, 3, 6, 9, or $12 \mathrm{~d}$. Expression analysis revealed that $\mathrm{HvCHS1}$ expression increased significantly at Day 3 and then decreased to Day 12, whereas $H v C H I$ and $H v O G T I$ expression peaked on either Day 1 

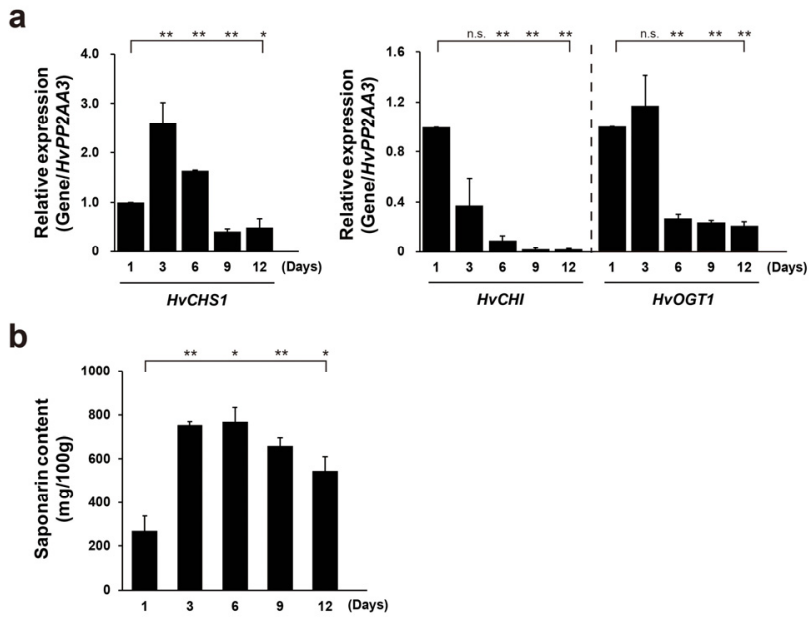

Fig. 2 Saponarin content and related gene expression in young barley seedlings. (a) Expression levels of $\mathrm{HvCHS1}, \mathrm{HvCHI}$, and HvOGT1. Barley plants grown at $23{ }^{\circ} \mathrm{C}$ under LD conditions were harvested at 1, 3, 6, 9 and $12 \mathrm{~d}$, and expression levels of $H v C H S 1, H v C H I$ and $H v O G T 1$ in each sample were analyzed using RT-qPCR. The expression levels of each gene at Day 1 were set to 1 . Values and error bars indicate mean \pm SEM values $(n=3)$. Asterisks indicate significant differences, when compare to the expression level at Day 1 (Student's $t$-test, ${ }^{*} P<$ $0.05,{ }^{* *} P<0.01$ ). n.s., not significant. (b) Saponarin content of young barley seedlings. The accumulation of saponarin was determined by UHPLC analysis

or Day 3 days and then decreased rapidly to Day 12 (Fig. 2a). These results are consistent with previous reports that $C H S$ and $\mathrm{CHI}$ expression are developmentally regulated (Schulz and Weissenböck 1988) and that CHS protein is present in young rice leaves but not in old leaves (Reddy et al. 1996).

The saponarin contents of young barley seedling extracts were also investigated. UHPLC analysis revealed that saponarin content highly accumulated at 3 and $6 \mathrm{~d}$ and slightly reduced until 9 and $12 \mathrm{~d}$ (Fig. 2b). These results indicated that higher expression of three saponarin biosynthetic genes at 1 or $3 \mathrm{~d}$ caused high accumulation of saponarin during early developmental stages of barley seedlings.

Effect of photoperiod on saponarin biosynthesis and accumulation

Because light/dark conditions have been reported to affect CHS and CHI expression (Dao et al. 2011; Wang et al. 2012) and because light is important for inducing or regulating plant metabolism (Yang et al. 2018), 15-d-old barley seedlings were transferred to a dark chamber for 1 or $2 \mathrm{~d}$, and then $H v C H S 1, H v C H I$, and $H v O G T 1$ expression were measured. Expression analysis revealed that $\mathrm{HvCHSl}$ and $\mathrm{HvCHI}$ expression decreased dramatically after the light/dark transition, whereas $H v O G T 1$ expression declined after $2 \mathrm{~d}$ under a
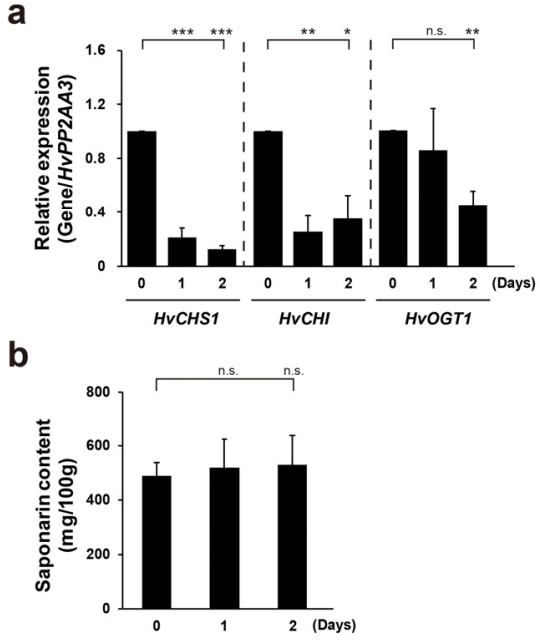

Fig. 3 Effect of light/dark transition on the saponarin content and related gene expression of young barley seedlings. (a) Expression levels of $H v C H S 1, H v C H I$, and $H v O G T 1$. Fifteen-d-old barley plants grown at $23^{\circ} \mathrm{C}$ under continuous light conditions were transferred to dark conditions for 1 or $2 \mathrm{~d}$, and the expression levels of $H v C H S 1, H v C H I$, and $H v O G T 1$ in each sample were analyzed using RT-qPCR. The expression levels of each gene at Day 0 were set to 1 . Values and error bars indicate mean \pm SEM values $(n=3)$. Asterisks indicate significant differences, when compared to the expression level at Day 0 (Student's $t$-test, ${ }^{*} P<0.05,{ }^{* *} P<0.01$ ). n.s., not significant. (b) Saponarin content of light-stressed barley seedlings. The accumulation of saponarin was determined by UHPLC analysis.

dark conditions (Fig. 3a). Many studies have reported that $\mathrm{CHS}$ and $\mathrm{CHI}$ promoters contain the CACGTG motif (i.e., G-box), which is associated with important light responses (Kaulen et al. 1986; Schulze-Lefert et al. 1989; Staiger et al. 1989), and other regulatory domains, such as the Box I, Box II, Box III, Box IV, or H-box (CCTACC) domains, which are also found in the $C H S$ promoter region, are also involved in light responses (Block et al. 1990; Lawton et al. 1990; Weisshaar et al. 1991). This result was consistent with the aforementioned findings.

The effect of light/dark transition on the saponarin contents of young barley seedling extracts was also investigated. However, the treatment had no effect on saponarin content (Fig. 3b). Given the finding that in vitro translation products of oat CHS mRNA are highly found at the dark or light phase (Knogge and Weissenbock 1986), it was expected that neither light nor dark would directly influence the translation of certain flavonoid biosynthetic genes, even if light exposure affects the expression of some flavonoid biosynthetic genes. These results indicate that the reduced $H v C H S 1, H v C H I$, and $H v O G T 1$ expression of the light-to-dark transitioned barley seedlings did not affect saponarin content, which suggests that $H v C H S 1, H v C H I$, and $H v O G T 1$ translation still occurs in darkness. 
a

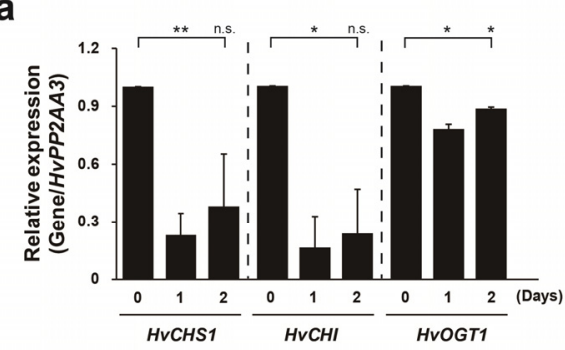

b

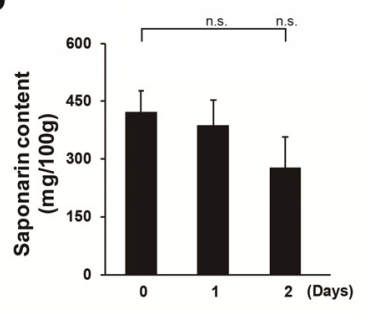

Fig. 4 Effect of drought on the saponarin content and related gene expression of young barley seedlings. (a) Expression levels of $H v C H S 1, H v C H I$, and HvOGT1. Fifteen-d-old barley plants grown at $23^{\circ} \mathrm{C}$ under long day (LD) conditions were subjected to dehydration conditions for 1 or $2 \mathrm{~d}$, and the expression levels of HvCHS1, $H v C H I$, and $H v O G T 1$ in each sample were analyzed using RTqPCR. The expression levels of each gene at Day 0 were set to 1 . Values and error bars indicate mean \pm SEM values $(n=3)$. Asterisks indicate significant differences, when compared to the expression level at Day 0 (Student's $t$-test, ${ }^{*} P<0.05,{ }^{* *} P<0.01$ ). n.s., not significant. (b) Saponarin content of drought-stressed barley seedlings. The accumulation of saponarin was determined by UHPLC analysis
Effect of abiotic stress on saponarin biosynthesis and accumulation

Because drought and temperature are the main environmental factors that affect the production of secondary metabolites, such as flavonoids, which are produced to cope with the oxidative stress generated by abiotic stresses (Yang et al. 2018), 15-d-old barley seedlings were exposed to dehydration conditions for 1 or $2 \mathrm{~d}$, and then $H v C H S 1, H v C H I$, and HvOGT1 expression were measured. Expression analysis revealed that $\mathrm{HvCHS1}$ and $\mathrm{HvCHI}$ expression decreased dramatically, whereas $H v O G T 1$ expression was unaffected (Fig. 4a). The saponarin content of the dehydrated barley seedlings was slightly lower at Day 2, although not significantly (Fig. 4b).

To investigate whether low or high temperatures affect saponarin content or saponarin biosynthetic gene expression, 15-d-old barley seedlings were transferred to low- or hightemperature conditions for 1 or $2 \mathrm{~d}$, and $H v C H S 1, H v C H I$, and $H v O G T 1$ were measured. The expression patterns of $H v C H S 1, H v C H I$, and $H v O G T 1$ were affected similarly by low- and high-temperature conditions. More specifically, $H v C H S 1$ and $H v C H I$ expression declined dramatically, whereas $H v O G T 1$ expression remained stable (Fig. 5a, b). However, neither low- nor high-temperature conditions significantly affected saponarin content (Fig. 5c, d). These a

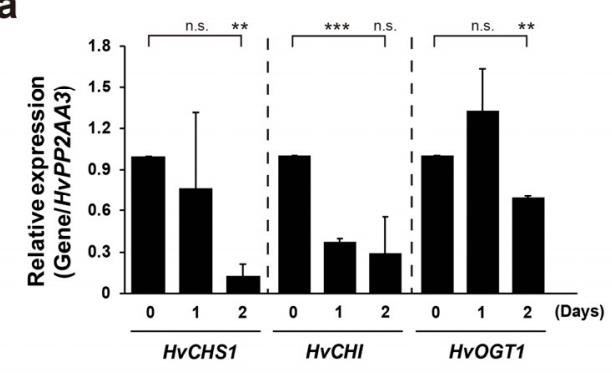

C

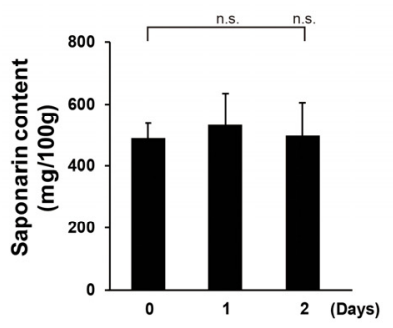

b

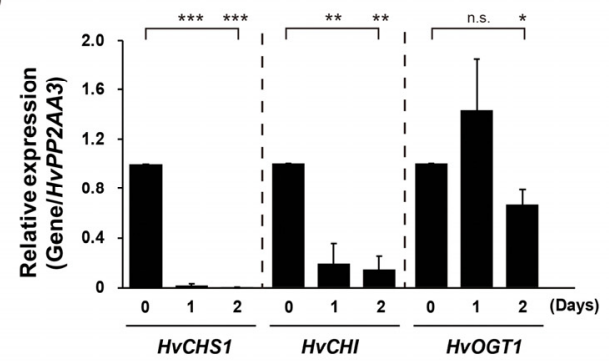

d

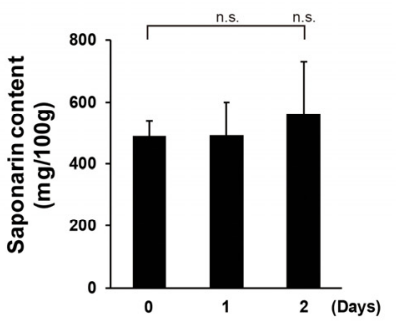

Fig. 5 Effect of low and high temperature on the saponarin content and related gene expression of young barley seedlings. (a and b) Expression levels of $\mathrm{HvCHS1}, \mathrm{HvCHI}$, and $\mathrm{HvOGT1}$. Fifteen-d-old barley plants grown at $23^{\circ} \mathrm{C}$ under long day (LD) conditions were transferred to low $\left(4^{\circ} \mathrm{C}\right.$, a) or high $\left(37^{\circ} \mathrm{C}\right.$, b) temperature conditions for 1 or $2 \mathrm{~d}$, and the expression levels of $H v C H S 1, H v C H I$, and $\mathrm{HvOGT1}$ in each sample were analyzed using RT-qPCR. The expression levels of each gene at Day 0 were set to 1 . Values and error bars indicate mean \pm SEM values $(\mathrm{n}=3)$. Asterisks indicate significant differences, when compared to the expression level at Day 1 (Student's $t$-test, $\left.{ }^{*} P<0.05,{ }^{*} P<0.01\right)$. n.s., not significant. (c and d) Saponarin content of low $\left(4^{\circ} \mathrm{C}\right.$, c) and high $\left(37^{\circ} \mathrm{C}\right.$, d) temperature-stressed barley seedlings. The accumulation of saponarin was determined by UHPLC analysis. 
results indicate that the reduced expression levels of $\mathrm{HvCHS1}$ and $H v C H I$, but not HvOGT1, were not correlated with saponarin accumulation under abiotic stress conditions.

Saponarin is synthesized from isovitexin (apigenin6- $C$ glucoside) after the addition of glucose in the 7-O position catalyzed by HvOGT as a soluble UDP-Glc:flavone glucosyltransferase (Blume et al. 1979). Because HvOGT catalyzes the final step of saponarin synthesis (Marinova et al. 2007), the stable expression of $\mathrm{HvOGTl}$ under abiotic stress conditions, such as drought and extreme temperature (Figs. 4 and 5), could maintain the level of saponarin content without significant change.

\section{Conclusions}

Flavonoids, such as saponarin, are used as medicinal ingredients and food additives and also served as plant secondary metabolites that provide protection against biotic and abiotic stresses. Even though saponarin mainly accumulates during early stages of barley leaf development, the correlation between saponarin content and the expression of saponarin biosynthetic pathway genes is not well understood. In the present study, the upregulation of $H v C H S 1, H v C H I$, and $H v O G T 1$ during early stages was associated with saponarin accumulation at later stages. In addition, exposure to abiotic stresses significantly affected the expression of $\mathrm{HvCHS} 1$ and $H v C H I$ but failed to affect either HvOGT1 expression or saponarin accumulation. Even though the expression levels of $H v C H S 1, H v C H I$, and $H v O G T 1$ under abiotic stress were not correlated with saponarin content, the results of the present study may provide a potential and profitable way to increase the accumulation of bioactive compounds, such as saponarin, in young barley seedlings.

\section{Acknowledgements}

This work was supported by a grant from the "Cooperative Research Program for Agriculture Science \& Technology Development (Project title: Identification and standardization of ameliorating effect on bioactive compounds for blood lipid levels from crop sprouts, Project No.: PJ01324902)", Rural Development Administration (RDA), Republic of Korea. We wish to express our gratitude towards Kyung Sook Chung, Hye Rim Shin, A Mi Yoon, Young Kwang Song, Wonyoung Park and Jae-Hyeok Park for their assistance.

\section{References}

Block A, Dangl JL, Hahlbrock K, Schulze-Lefert P (1990) Functional borders, genetic fine structure, and distance requirements of cis elements mediating light responsiveness of the parsley chalcone synthase promoter. Proc Natl Acad Sci USA 87: 5387-5391

Blume DE, Jaworski JG, McClure JW (1979) Uridinediphosphateglucose: Isovitexin 7-O-glucosyltransferase from barley protoplasts: Subcellular localization. Planta 146:199-202

Christie JM, Jenkins GI (1996) Distinct UV-B and UV-A/blue light signal transduction pathways induce chalcone synthase gene expression in Arabidopsis cells. Plant Cell 8:1555-1567

Cushnie TP, Lamb AJ (2005) Antimicrobial activity of flavonoids. Int J Antimicrob Agents 26:343-356

Dao TT, Linthorst HJ, Verpoorte R (2011) Chalcone synthase and its functions in plant resistance. Phytochem Rev 10:397-412

Faktor O, Kooter JM, Loake GJ, Dixone RA, Lamb CJ (1997) Differential utilization of regulatory cis-elements for stressinduced and tissuespecific activity of a French bean chalcone synthase promoter. Plant Science 124:175-182

Hartmann U, Sagasser M, Mehrtens F, Stracke R, Weisshaar B (2005) Differential combinatorial interactions of cis-acting elements recognized by R2R3-MYB, BZIP, and BHLH factors control light-responsive and tissue-specific activation of phenylpropanoid biosynthesis genes. Plant Mol Biol 57: 155-171

Hartmann U, Valentine WJ, Christie JM, Hays J, Jenkins GI, Weisshaar B (1998) Identification of UV/blue light-response elements in the Arabidopsis thaliana chalcone synthase promoter using a homologous protoplast transient expression system. Plant Mol Biol 36:741-754

Hertog MG, Feskens EJ, Hollman PC, Katan MB, Kromhout D (1993) Dietary antioxidant flavonoids and risk of coronary heart disease: the Zutphen Elderly Study. Lancet 342:1007-1011

Honsdorf N, March TJ, Berger B, Tester M, Pillen K (2014) High-throughput phenotyping to detect drought tolerance QTL in wild barley introgression lines. PLoS One 9:e97047

Hutzler P, Fischbach R, Heller W, Jungblut T, Reuber S, Schmitz R, Veit M, Weissenbo“ck G, Schnitzler J (1998) Tissue localization of phenolic compounds in plants by confocal laser scanning microscopy. J Exp Bot 49:953-965

Janska A, Hodek J, Svoboda P, Zamecnik J, Prasil IT, Vlasakova E, Milella L, Ovesna J (2013) The choice of reference gene set for assessing gene expression in barley (Hordeum vulgare L.) under low temperature and drought stress. Mol Genet Genomics 288:639-649

Jende-Strid B (1993) Genetic control of flavonoid biosynthesis in barley. Hereditas 119:187-204

Kamiyama M, Shibamoto T (2012) Flavonoids with Potent Antioxidant Activity Found in Young Green Barley Leaves. $J$ Agric Food Chem 60:6260-6267

Kaulen H, Schell J, Kreuzaler F (1986) Light-induced expression of the chimeric chalcone synthase-NPTII gene in tobacco cells. EMBO J 5:1-8 
Knogge W, Weissenbock G(1986) Tissue-distribution of secondary phenolic biosynthesis in developing primary leaves of Avena sativa L. Planta 167:196-205

Lawton MA, Clouse SD, Lamb CJ (1990) Glutathione-elicited changes in chromatin structure within the promoter of the defense gene chalcone synthase. Plant Cell Rep 8:561-564

Lepiniec L, Debeaujon I, Routaboul JM, Baudry A, Pourcel L, Nesi N, Caboche M (2006) Genetics and biochemistry of seed flavonoids. Annu Rev Plant Biol 57:405-430

Marinova K, Kleinschmidt K, Weissenbock G, Klein M (2007) Flavonoid biosynthesis in barley primary leaves requires the presence of the vacuole and controls the activity of vacuolar flavonoid transport. Plant Physiol 144:432-444

Middleton E, Jr. (1998) Effect of plant flavonoids on immune and inflammatory cell function. Adv Exp Med Biol 439:175-182

Mojzisova G, Mirossay L, Kucerova D, Kyselovic J, Mirossay A, Mojzis J (2006) Protective effect of selected flavonoids on in vitro daunorubicin-induced cardiotoxicity. Phytother Res 20: 110-114

Moses T, Papadopoulou KK, Osbourn A (2014) Metabolic and functional diversity of saponins, biosynthetic intermediates and semi-synthetic derivatives. Crit Rev Biochem Mol Biol 49:439-462

Peukert M, Weise S, Roder MS, Matthies IE (2013) Development of SNP markers for genes of the phenylpropanoid pathway and their association to kernel and malting traits in barley. BMC Genetics 14:16

Reddy AR, Scheffler B, Madhuri G, Srivastava MN, Kumar A, Sathyanarayanan PV, Nair S, Mohan M (1996) Chalcone synthase in rice (Oryza sativa L.): detection of the CHS protein in seedlings and molecular mapping of the chs locus. Plant Mol Biol 32:735-743

Reuber S, Bornman JF, Weissenbo“ck G (1996) A flavonoid mutant of barley (Hordeum vulgare L.) exhibits increased sensitivity to UV-B radiation in the primary leaf. Plant Cell Environ 19:593-601

Reuber S, Jende-Strid B, Wray V, Weissenbock G (1997) Accumulation of the chalcone isosalipurposide in primary leaves of barley flavonoid mutants indicates a defective chalcone isomerase. Physiol Plant 101:827-832

Schulz M, Weissenbo“ck G (1986) Isolation and separation of epidermal and mesophyll protoplasts from rye primary leaves: tissue specific characteristics of secondary phenolic product accumulation. Z Naturforsch [C] 41:22-27
Schulz M, Weissenbo“ck G (1988) Dynamics of the tissue-specific metabolism of luteolin glucuronides in the mesophyll of rye primary leaves (Secale cereale). Z Naturforsch [C] 43:187-193

Schulze-Lefert P, Becker-Andre M, Schulz W, Hahlbrock K, Dangl JL (1989) Functional architecture of the light-responsive chalcone synthase promoter from parsley. Plant Cell 1:707-714

Seo KH, Park MJ, Ra JE, Han SI, Nam MH, Kim JH, Lee JH, Seo WD (2014) Saponarin from barley sprouts inhibits NF-kappaB and MAPK on LPS-induced RAW 264.7 cells. Food Funct 5:3005-3013

Seo WD, Yuk HJ, Curtis-Long MJ, Jang KC, Lee JH, Han SI, Kang HW, Nam MH, Lee SJ, Lee JH, Park KH (2013) Effect of the growth stage and cultivar on policosanol profiles of barley sprouts and their adenosine 5'-monophosphate-activated protein kinase activation. J Agric Food Chem 61:1117-1123

Staiger D, Kaulen H, Schell J (1989) A CACGTG motif of the Antirrhinum majus chalcone synthase promoter is recognized by an evolutionarily conserved nuclear protein. Proc Natl Acad Sci USA 86:6930-6934

Wang W, Wang H-L, Wan S-B, Zhang J-H, Zhang P, Zhan J-C, Huang W-D (2012) Chalcone isomerase in grape vine: gene expression and localization in the developing fruit. Biologia Plantarum 56:545-550

Weisshaar B, Armstrong GA, Block A, da Costa e Silva O, Hahlbrock K (1991) Light-inducible and constitutively expressed DNA-binding proteins recognizing a plant promoter element with functional relevance in light responsiveness. EMBO J 10:1777-1786

Weisskopf L, Abou-Mansour E, Fromin N, Tomasi N, Santelia D, Edelkott I, Neumann G, Aragno M, Tabacchi R, Martinoia E (2006) White lupin has developed a complex strategy to limit microbial degradation of secreted citrate required for phosphate acquisition. Plant Cell Environ 29:919-927

Wingender R, Rohrig H, Horicke C, Wing D, Schell J (1989) Differential regulation of soybean chalcone synthase genes in plant defence, symbiosis and upon environmental stimuli. Mol Gen Genet 218:315-322

Yang L, Wen KS, Ruan X, Zhao YX, Wei F, Wang Q (2018) Response of Plant Secondary Metabolites to Environmental Factors. Molecules 23

Zhang L, Zhang Q, Jiang Y, Li Y, Zhang H, Li R (2018) Reference genes identification for normalization of qPCR under multiple stresses in Hordeum brevisubulatum. Plant Methods 14:110 